\title{
Salvia Hispanica L (Chia Seeds) as Brain Superfood - How Seeds Increase Intelligence
}

\author{
Peter Onneken ${ }^{1}$ \\ ${ }^{1}$ Head of Research, Institute of Diet and Health, Cologne, Germany \\ Correspondence: Peter Onneken, Head of Research, Institute of Diet and Health, Cologne, Germany. E-mail: \\ research@instituteofdiet.com
}

Received: April 4, 2018 Accepted: May 10, 2018 Online Published: June 11, 2018

doi:10.5539/gjhs.v10n7p69

URL: https://doi.org/10.5539/gjhs.v10n7p69

\begin{abstract}
The influence of nutrition on cognitive abilities is undisputed in academic literature. Omega- 3 fatty acids in particular delivered convincing results in several studies. In recent years chia seeds (Salvia Hispanica L) are becoming increasingly popular in Europe as well. In a trial the authors divided the study participants into two groups: an intervention group and a control group. The participants of the intervention group consumed a daily dose of 5 grams chia seeds over a time period of 21 days.

In total, the test group that participated in the intervention performed significantly better in the retest than the comparison group. They improved their verbal intelligence and enhanced their ability in problem solving. Insofar, the classification of Salvia Hispanica $L$ as a superfood, respectively brain superfood, is scientifically justified.
\end{abstract}

Keywords: brain superfood, Omega-3 fatty acids, Salvia Hispanica $L$

\section{Background}

The influence of nutrition on cognitive abilities is undisputed in academic literature (Zhang, Hebert, \& Muldoon, 2005). Numerous studies have shown that the effects of polyunsaturated fats and other foods, such as milk, meat or oils are in fact measurable (Neumann, Murphy, Gewa, Grillenberger, \& Bwibo, 2007).

Omega-3 fatty acids in particular delivered convincing results in several studies. There is justified assumption that with an according diet a) the risk of developing Alzheimer's can be reduced and b) cognitive performance can be increased. Hooper et al. have recently proven that verbal intelligence decreased less if omega-3 fatty acids were taken in (Hooper et al., 2017). Even though other studies could not spot a significant connection between omega-3 fatty acids and the reduction of Alzheimer's specific symptoms (Andrieu et al., 2017). Mazza, Pomponi, Janiiri, et al. have found that omega-3 fatty acids play a crucial role in the activity of the nervous system, cognitive development, memory-related learning and the development of brain synapses. The review verifies the effect of omega-3 fatty acids on the anatomical and functional development of the central nervous system (Pomponi, Janiri, Bria, \& Mazza, 2007). In addition, the studies prove that the omega-3 fatty acid decosahexaenoic acid can be of great benefit in preventing neuronal diseases and supporting mental health (Wu, Yin, \& Gomez, 2008).

\subsection{Salvia Hispanica L as a Source of Omega-3}

In recent years, chia seeds (Salvia hispanica L) have becoming increasingly popular in Europe. The European Union (EU) approved them as a so-called "novel food". The seeds consist of about $25-40 \%$ oil and are composed of about $60 \% \omega-3$ alpha-linolenic acid (ALA) and 20\% $\omega-6$ linolenic acid. The fatty acids are required by the body to stay healthy and cannot be produced synthetically (Mohd Ali, Yeap, Ho, Beh, Tan, \& Tan, 2012). The chia seeds consist of proteins (15-25\%), fats (30-33\%), carbohydrates (26-41\%), fibre (18-30\%), ash (4-5\%), minerals, vitamins and contain a high proportion of antioxidants (Ixtaina, Nolasco, \& Tomas, 2008).

Studies on chia seeds show a correlation between a) the consumption of predominantly monounsaturated fatty acids and a low intake of polyunsaturated fatty acids and b) the occurrence of cardiovascular diseases, diabetes and metabolic syndrome (Martha et al., 2012). Fernandez et al. have discovered additional medical advantages of chia seeds, which not only cause the reduction of cholesterol and the inhibition of blood clotting but also may prevent stress and epilepsy. Furthermore, research assumes that the consumption of chia seeds during pregnancy can improve the development of the foetus' brain and retina (Fernandez, Vidueiros, Ayerza, Coates, \& Pallaro, 2008). So far there hasn't been much research in the favourable effects of an intake of chia seeds on schoolchildren, 
students or Alzheimer's patients. Therefore, this study intends to examine the effects of Salvia hispanica $L$ on cognitive abilities by means of a chia intervention.

\section{Methodology}

The study is based on the evaluation of tests that measure cognitive performance. They were conducted with students of the Technical University at Dortmund, Germany in the summer of 2017. The order of study distinguished between an intervention group and a control group. The tests were repeated once. In the meantime, the intervention with chia seeds was conducted. Besides the on-site testing, the probands were requested to complete additional tasks.

\subsection{Study Participants}

From a total of 34,600 students, two groups were chosen to participate in the study. The participants were 21.3 years old on average and $96 \%$ female. The high percentage of female participants deliberately does not represent the average population, but is oriented towards the significantly higher proportion of Alzheimer's cases attributed to the longer life expectancy of women. None of the participants suffered from allergies, hypertension/hypotension or took blood thinners. One test person stated she was chronically ill.

\subsection{Randomisation}

Following the pre-test, which particularly inquired about health risks in order to prevent the possible blood thinning effect caused by a chia overdose, the participants were randomly selected into two groups.

\subsection{Interventions/Measures}

The participants were divided into two groups: an intervention group and a control group. The participants of the intervention group consumed a daily dose of 5 grams chia seeds over a time period of 21 days. The control group continued to eat as before. The authors of the study consciously chose a low dosage. The European Food Safety Authority (EFSA) approved chia seeds as food in 2009. The authority recommends a maximum daily dose of 15 grams. The participants were explicitly requested to take these guidelines seriously and to only consume the lower chia seed dosage of 5 grams per day.

\subsection{Method of Testing}

After the devision of the subjects into the according groups, several tests were performed on-site, which aimed to examine the participants cognitive abilities (in particular memory performance). Additionally, verbal intelligence was tested by means of the sentence recognition test of the Clinic of Psychiatry and Psychotherapy at the Ludwig Maximilian University in Munich, Germany. The probands were requested to answer 31 questions of the "sentence recognition test" in the category "difficult". Furthermore, the participants had to assemble a LEGO airplane consisting of 111 pieces. Thereby, the time was measured from start to completion of the airplane. The correlation between the ability to assemble LEGO bricks and intelligence was demonstrated by Richardson, Hunt and Richardson (M. Richardson, Hunt, \& C. Richardson, 2014). The retest enabled us to measure how well the work steps had been memorised and, thus, revealed memory performance.

Moreover, a classical memory test was conducted. The participants were asked to memorise 14 terms within one minute. A first test was run after 60 minutes: the probands were asked to write down the memorised terms.

All tests were repeated after an intervention period of three weeks.

\section{Results}

In total, the test group that participated in the intervention performed significantly better in the retest than the comparison group.

\subsection{Memory Test}

As expected, both the probands of the intervention group and probands of the comparison group could remember less terms after three weeks than after the initial query made after one hour. At first, both group participants behaved nearly identically. The subjects of the intervention group remembered 7.9 terms on average, and the subjects of the control group remembered 7.6 terms on average. After three weeks, the difference was clearly visible. The control group, whose participants had not changed their diet, could only remember 5.4 terms on average. The intervention group, whose participants had consumed chia seeds, could remember 6.6 terms on average. This means that the intervention group only forgot $16.5 \%$ of the memorised terms, while the control group forgot almost twice as much, namely $29 \%$.

However, with a p-value of 0.74 , the testing cannot be rated as statistically significant. 


\subsection{Sentence Completion Test}

As the degree of difficulty of the sentence completion test was very high, both test groups could only answer 7.0 (intervention group) and 6.4 questions (control group) correctly on average. Similar to the memory test, the difference increased enormously after the intervention period - now the intervention group could answer 6.3 questions correctly, and the control group only 2.6 questions on average. This means that the verbal intelligence of the intervention group increased $142 \%$ compared with the control group. If converting these results analogously to the test methods of the University of Munich, they show an increase of the verbal intelligence quotient from 106.3 vlQ to $118.0 \mathrm{vlQ}$. In comparison, the verbal intelligent quotient of the control group increased from $102.7 \mathrm{vlQ}$ to $112.9 \mathrm{vlQ}$.

With a p-value of 0.037 , the testing is rated as statistically significant.

\subsection{Applied Intelligence/LEGO Testing}

The assembly of the 111-part aircraft took 41 minutes and 41 seconds on average during the first testing. Both groups completed the assembly in nearly the same time, with only a few minutes difference. And expectedly, both groups managed to improve due to the repetition effect. Yet, the intervention group performed much better than the control group - now the intervention group assembled the airplane 14 minutes and 59 seconds faster on average. The control group was only 2 minutes and 31 seconds faster.

This test series has a significance of $p=0.048$, hence the effect is highly probable.

\section{Discussion}

In the authors view, the basic assumption is confirmed that a chia-rich nutrition may have positive effects on cognitive abilities. This was proven in particular not only by the two tests with high significance but also by the memory task, which indicates that a dietary intake of Salvia hispanica $L$ can positively impact memory abilities.

The authors were notably surprised by the significant differences in the progress of learning between the test groups. Further studies should investigate if these results can be confirmed in the long term. With all appropriate scientific caution, it appears to be evident thus far that in an aging society, dealing with the increase of cognitive diseases such as Alzheimer's or dementia, the daily consumption of Salvia hispanica $L$ is highly recommendable.

The examined test group consisted of young adults. All participants were still studying at a university level. Future research should place an emphasis on schoolchildren in order to evaluate if the positive results demonstrated by the chia group can be improved in younger ages, especially before puberty. The authors are convinced by now that a valid statement can be made that the intake of Salvia hispanica $L$ leads, with high significance, to an improvement of applied intelligence and verbal intelligence. Insofar, the classification of Salvia hispanica L as a "superfood", respectively brain superfood, seems to be scientifically justified.

\section{Competing Interests Statement}

The author declares that there are no competing or potential conflicts of interest.

\section{References}

Andrieu, S., Guyonnet, S., Coley, N., Cantet, C., Bonnefoy, M., Bordes, S., ... \& Desclaux, F. (2017). Effect of long-term omega 3 polyunsaturated fatty acid supplementation with or without multidomain intervention on cognitive function in elderly adults with memory complaints (MAPT): a randomised, placebo-controlled trial. The Lancet Neurology, 16(5), 377-389. https://doi.org/10.1016/S1474-4422(17)30040-6

Fernandez, I., Vidueiros, S. M., Ayerza, R., Coates, W., \& Pallaro, A. (2008). Impact of chia (Salvia hispanica L. (on the immune system: preliminary study. The Proceedings of the Nutrition Society, 67(OCE). https://doi.org/10.1017/S0029665108006216

Guevara-Cruz, M., Tovar, A. R., Aguilar-Salinas, C. A., Medina-Vera, I., Gil-Zenteno, L., Hernández-Viveros, I., ... \& Torres, N. (2011). A Dietary Pattern Including Nopal, Chia Seed, Soy Protein, and Oat Reduces Serum Triglycerides and Glucose Intolerance in Patients with Metabolic Syndrome-. The Journal of nutrition, 142(1), 64-69.

Hooper, C., de Souto Barreto, P., Coley, N., Cantet, C., Cesari, M., Andrieu, S., ... \& MAPT/DSA Study Group. (2017). Cognitive changes with omega-3 polyunsaturated fatty acids in non-demented older adults with low omega-3 index. The journal of nutrition, health \& aging, 21(9), 988-993.

Ixtaina, V. Y., Nolasco, S. M., \& Tomas, M. C. (2008). Physical properties of chia (Salvia hispanica L.) seeds. Industrial crops and products, 28(3), 286-293. 
Mazza, M., Pomponi, M., Janiri, L., Bria, P., \& Mazza, S. (2007). Omega-3 fatty acids and antioxidants in neurological and psychiatric diseases: an overview. Progress in Neuro-Psychopharmacology and Biological Psychiatry, 31(1), 12-26.

Mohd Ali, N., Yeap, S. K., Ho, W. Y., Beh, B. K., Tan, S. W., \& Tan, S. G. (2012). The promising future of chia, Salvia hispanica L. BioMed Research International, 2012. https://doi.org/10.1155/2012/171956

Neumann, C. G., Murphy, S. P., Gewa, C., Grillenberger, M., \& Bwibo, N. O. (2007). Meat supplementation improves growth, cognitive, and behavioral outcomes in Kenyan children. the Journal of Nutrition, 137(4), 1119-1123.

Richardson, M., Hunt, T. E., \& Richardson, C. (2014). Children's Construction Task Performance and Spatial Ability: Controlling Task Complexity and Predicting Mathematics Performance. Perceptual and motor skills, 119(3), 741-757.

Test on verbal intelligence of the Technische Universität München, Retrieved from http://psytest.psy.med.uni-muenchen.de/tests.htm

Wu, A., Ying, Z., \& Gomez-Pinilla, F. (2008). Docosahexaenoic acid dietary supplementation enhances the effects of exercise on synaptic plasticity and cognition. Neuroscience, 155(3), 751-759.

Zhang, J., Hebert, J. R., \& Muldoon, M. F. (2005). Dietary fat intake is associated with psychosocial and cognitive functioning of school-aged children in the United States. J Nutr., 135(8), 1967-1973.

\section{Copyrights}

Copyright for this article is retained by the author(s), with first publication rights granted to the journal.

This is an open-access article distributed under the terms and conditions of the Creative Commons Attribution license (http://creativecommons.org/licenses/by/4.0/). 